\title{
8
}
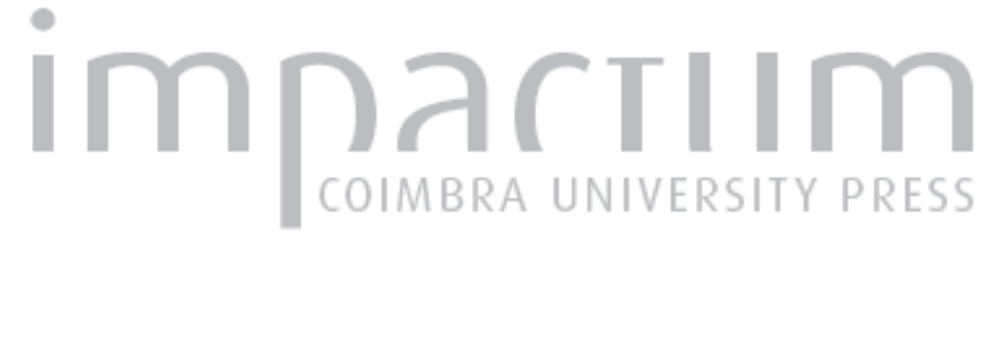

\section{O serviço de saúde nos bombeiros: a sua importância da univítima à Medicina de catástrofe} \author{
$\begin{array}{ll}\text { Autor(es): } & \text { Bandeira, Romero; Reis, Ana Mafalda; Leão, Rui Ponce; Gandra, Sara; } \\ \text { Gandra, Romero }\end{array}$ \\ Publicado por: Associação Portuguesa de Riscos, Prevenção e Segurança \\ URL \\ persistente: \\ URI:http://hdl.handle.net/10316.2/36230 \\ DOI: \\ DOI:http://dx.doi.org/10.14195/1647-7723_14_10 \\ Accessed : $\quad$ 26-Apr-2023 11:04:44
}

A navegação consulta e descarregamento dos títulos inseridos nas Bibliotecas Digitais UC Digitalis, UC Pombalina e UC Impactum, pressupõem a aceitação plena e sem reservas dos Termos e Condições de Uso destas Bibliotecas Digitais, disponíveis em https://digitalis.uc.pt/pt-pt/termos.

Conforme exposto nos referidos Termos e Condições de Uso, o descarregamento de títulos de acesso restrito requer uma licença válida de autorização devendo o utilizador aceder ao(s) documento(s) a partir de um endereço de IP da instituição detentora da supramencionada licença.

Ao utilizador é apenas permitido o descarregamento para uso pessoal, pelo que o emprego do(s) título(s) descarregado(s) para outro fim, designadamente comercial, carece de autorização do respetivo autor ou editor da obra.

Na medida em que todas as obras da UC Digitalis se encontram protegidas pelo Código do Direito de Autor e Direitos Conexos e demais legislação aplicável, toda a cópia, parcial ou total, deste documento, nos casos em que é legalmente admitida, deverá conter ou fazer-se acompanhar por este aviso. 
(Página deixada propositadamente em branco) 
pp. $97-106$

\title{
O SERVIÇO DE SAÚDE NOS BOMBEIROS. A SUA IMPORTÂNCIA, DA UNIVÍTIMA À MEDICINA DE CATÁSTROFE
}

\author{
Romero Bandeira*, Ana Mafalda Reis**, Rui Ponce Leão**, Sara Gandra*** e Romero Gandra**** \\ * Instituto de Ciências Biomédicas Abel Salazar (ICBAS) , Univiversidade do Porto, \\ Coordenador do Mestrado em Medicina de Catástrofe, Comandante $\mathrm{QH}$ \\ ** Instituto de Ciências Biomédicas Abel Salazar (ICBAS) , Universidade do Porto \\ *** Hospital Geral de Santo António, Porto \\ **** Escola Nacional de Bombeiros e Bombeiros Voluntários de S. Pedro da Cova
}

\section{RESUMO}

Desde 1951, as contomos do Serviço de Saúde evoluíram na medida em que as solicitações para socorro a pessoas aumentaram exponencialmente, ao mesmo tempo que os bombeiros concluíram que, para ser exercida com competência, a prestação de cuidados exigia que eles próprios tivessem tanto uma formação, como um perfil físico e psicológico adequados. Dada a vasticão do tema, os autores qptaram por focal izar o seu trabalho nas vertentes que consideram de qualidade major - Medicina Ocupacional, Medicina no Terreno e Formação.

Palavras chave: Saúde, bonbeiros, catástrofe, imagiologia, formação.

\section{ABSTRACT}

Since 1951, the characteristics of Health Services developed as solicitations to help people grew exponentionally. At the same time, firemen concluded that, in order to do their job with competence, theymust have an adequate formation and an adequate physical and psychological profile. The authors focal ized their work on the questions they consider of a major quality - Ocapational Medicine, Field Medicine and Formation.

Key words: Health, firemen, disaster, imageology, formation.

\section{RESUMÉ}

Depuis 1951, les caractéristiques des Services Sanitaires ont évoluéà la mesure de l'accroissement exponentiel des demandes de secours. En même temps, pour que sa mission puisse être exercée avec compétence, les pampiers ant vérifié qu' ils doivent posséder formation et profil physique et psychologique spécifiques. Devant l'extension du thère les auteurs ont décidé une focalisation des son travail sur les questions qu' il ls considèrent d'une quali té majeure - Nédicine Ocaupationnelle, Médicine de Terrain et Formation.

Mbts clé: Santé, pompiers, catastrophe, imageologie, formation. 


\section{Introdução}

Os Corpos de Bombeiros encontram-se uniformemente estruturados desde o paradigmático Decreto-Lei n 38.439 de 27 de Setembro de 1951 (Cristiano-Santos, 1995) em 3 serviços: Incêndio, Saúde e Socorros a Náufragos. Ao longo do tempo ficou evidenciadb que esta estntura, muito simples, radicava em si própria, as necessidades prioritárias que as populações dos Municípios reclamavam em termos de socorro.

Um das exemplos mais evidentes é o das socorros a Núufragos; desenvolvido a partir das necessidades colocadas por uma navegação de cabotagem, evoluiu, afastando-se hoje do litoral marítimo, para locais recânditos do interior, acnde se toma necessário o socorro fluvial por vezes através de equipas de mergulhadores. Quer em acidentes extrínsecos, que se acabam por projectar nos cursos de água (GNRRA, 2005) quer emacidentes intrínsecas aas mesmos, por exemplo nos designados desportos de aventura, os quais frequentemente obrigam à mobilização extraordinária de meios que no passado não eram sequer equacionados, mas que se enquadram naquela estrutura tão simples, quanto eficaz, de há tantos anos.

Porém, foi o serviço de saúde que se viu dbrigado a fazer uma adaptação constante das suas capacidades, dando respostas a situações que the foram sendo colocadas a nível do serviço de urgência extra-hospitalar, fruto, não só, da evolução dos conhecimentos científicos nesta área mas também da tecnologia concomitante que surgiu neste âmbito.

O serviço criado ao tempo (Áostnno, 2005), quer nos corpos de bombeiros mais evoluídos, quer nos mais modestos, desde o litoral ao interior do País, confinava-se basicamente a auto-macas, servidas por guamições que procuravam a mprir cabalmente o seu dever através de vicissitudes múltiplas.

No entanto, há que relevar que muitos corpos de Bombeiros possuíam um número elevado de médicos, enfermeiros e farmacêticicas, a que se associavam os maqueiros que eram recrutados entre aqueles que, pelos mais variados motivos, estavam vocacionados para o serviço de saúde.

Hoje em dia, a urgência extra-hospitalar, não se encontra confinada exclusivamente ao serviço de saúde dos conpos de bombeiros, existindo a acção no terreno do Instituto Nacional de Emergência Médica, que possui as competências que de toobs são conhecidas. No entanto, torna-se extraordinariamente usual a articulação de uma UMER com uma ABSC dos corpos de bombeiros, no socorro às vítimas de doença ou acidente com as competentes coordenações do CDOS e CODU.

\section{Evolução Conceptual}

Desde 1951, os contomos do Serviço de Saúde evoluíram, na medida em que as solicitações para socorro a pessoas aumentaram exponencialmente, ao mesmo tempo que os bombeiros concluíram que, não só a prestação de cuidados para ser exercida com competência exigia que eles próprios tivessem a formação adequada, mas também que o perfil físico e psíquico do bonbeiro fosse o desejável.

Assim, em termos funcionais, tornar-se-ia necessário que o Serviço de Saúde assumisse as vertentes da medicina ocupacional, da formação e da intervenção. Por despacho de 30 de Setembro de 1982, do então Presidente do novel Serviço Nacional de Bombeiros, Padre Dr. Victor Melicias, foi ao primeiro autor deste artigo cometida a função de elaborar uma proposta para funcionamento do referido Serviço da Saúde, documento, em anexo, tendo como co-autor Eduardo Agostinho, médico, ao tempo Comandante das Bambeiros Voluntários de Rio Mbior.

Mais tarde, a 10 de Maio de 1995 surgiu uma proposta de trabalho da Comissão de Saúde do SNB/ LBP. Posteriormente, um outro documento foi elaborado a 10 de Novembro de 1999 tendo igualmente como autores, Romero Bandeira, Dra. Céu Teiga e Enfermeira Sara Gandra, dando assim cumprimento ao solicitado pela Inspecção Superior de Banbeiros através de ofícios de 13 e 14 de attubro de 1999.

A entrada no $3^{\circ}$ Millénio não se compadece com o diferir no espaço e no tempo de soluções que podem optimizar a qualidade de vida, quer de vítimas, quer dos intervenientes. Assim, e dada a vasticăo do tema, os autores optaram por focalizar o seu trabalho nas vertentes major: Medicina Ocupacional, Medicina no Terreno e Formação.

\section{Medicina Ocupacional}

Nesta ordem de ideias a Medicina Ocupacional deve ser una pedra angular de todb este edifício: se "quem não sabe não salva, nem se salva" (GnNRA, 2007) obviamente que quem estiver incapacitado psíquica ou fisicamente também não. Recentemente em artigo publicado no The New England Joumal of Medicine (Kares et al., 2007), intituladb "Energency Duties and Deaths from Heart Disease among Firefighters in the Lnited States" extratamos do abstract: "Background: Heart disease causes 45\% of the deaths that ocur among U.S. Fire-fighters while they are on duty. We examined duty-specific risks of death from corcuary heart disease among on-duty U.S. firefighters from 1994 to 2004. 
Conclusions: Certain energency fire fighting aties were associated with a risk of death from coronary heart disease that was markedly higher than the risk associated with nonemergency duties. Fire suppression was associated with the highest risk, which was approximately 10 to 100 times as high as that for nonemergency duties."

Por outro lado Benowitz (2007) indica uma classificação de doenças cardiovasculares de possiveis causas tóxicas evidencianob vários tipos de patologia, conforme a seguir se explicita:

"Cardiac arnhytmia: Arsenic, chlorofluorocarbon propellants, Hydrocarbon solvents (e.g. , 1,1,1thrichloroethane and thrichloroethylene), Organophosphate and carbamate insecticides.

Coronary artery disease: Air pollution, Carbon disulfide, Carbonmonoxide, Lead(?) .

Hypertension: Cadmium, Carbon disulfide, Lead.

Myocardial asphyxiation: Carbon monoxide, Cyanide, Hydrogen sulfide.

Myocardial injury: Antimony, Arsenic, Arsine, Cobalt, Lead.

Nonatheramatous ischemic heart disease: Organic nitrates (e.g., nitroglycerin and ethylene glycol diritrate)

Peripheral arterial occlusive disease: Arsenic, Cadmium, Lead."

Para além de situações tóxicas agressivas menos frequentes, os Bombeiros podem sofrer intoxicação aguda pelo co (Monóxido de Carbono) , ou mesmo morte, como nos casos dos falecimento em serviço de bombeiros dos Corpos de Santa Marta de Penaguião e Porto de Mós

Porémna intervenção repetida ao longo de vários dias, semanas ou meses, a intoxicação poder-se-á tomar crónica.

A terapêutica com Oxigénio Hiperbárico (a pressão superior à pressão atmosférica) é hipótese terapêtica a ter en consideração; trata-se da mesma técnica que éuti i izada nos acidentes de mergulho.

Curiosamente, o trabalho dos Bombeiros e o Mergulho estão ligados, entre autros, pela história do "Capacete de Mergulho": em 1823, Charles Deane construiu um "Capacete de Frmo" destinado a ser utilizado pelos Bombeiros no combate aos fogos; porém, sendo construído em metal, aquecia imenso, devido às elevadas temperaturas em que o Bombeiro se movimentava. Face ao perigo daí resultante e às experiências efectuadas verificcu-se que o "Capacete de Fumo" não era utilizável para as funções para as quais tinha sido idealizado. Tentando não perder 0 investimento efectuado, adaptou-o a "Capacete de Mergulho", antecessor dos actuais capacetes de mergulho (Brwson B., 2005) .

atrras profissões estão também sujeitas a este tipo de intoxicação, quer no caso de acidente por perturbação brusca do normal funcionamento dos maquinismos que utilizam quer por má ventilação do local, podendo ser vítimas de intoxicação aguda au crónica.

Estão também descritos casos de suicídio e homicídio pelo mesmo agente etiológico.

O cadáver por exemplo: rigidez cadavérica menos intensa, de menor duração, surginab mais tarde, a cor da face tem similitude com a de um individuo vivo, existinob uma cor mais clara nas zonas de hipostase, os órgãos internos adquirem um tom carmim, o sangue tem cor rósea e está fluido, a putrefacção inicia-se mais tardiamente (CALAEIG, 2004; FRANA̧A, 2004).

As investigações científicas sobre a intoxicação pelo co têm uma fase determinante, na segunda metade do século XIX com um investigador, J. S. Haldane, escocês nascido em 1860 e professor de Fisiologia em Oxford, que, preocupado com a morte de mineiros resultante de contactarem com Monóxido de Carbono durante o trabalho, efectuou experiências em que ele era a sua própria cobaia, intoxicando-se metodicamente e retirando o seu sangue para determinar o grau de intoxicação (Brwsor, 2005) .

o Monóxido de Carbono, quando a 1 atmosfera (pressão atmosférica "normal") , é um gás incolor e inodoro, não irritante, com densidade de $0.967 \mathrm{em}$ relação ao ar e massa volúmica de 1,25g/litro. Tem ponto de fusão a - $199^{\circ}$ Celsius e ponto de ebulição a -195, $5^{\circ}$ Celsius. É inflamável e de grande toxicicade sendo, nomeadamente, produzido pela combustão de combustíveis com carbono na sua composição.

Para além do Monóxido de Carbono exógeno, que está a ser abordado neste texto, o organismo produz CO no catabolismo do "heme" resultando na saturação de 0,5\% da hemogldoina no sangue venoso.

Tal como o óxido nítrico, também o Monóxido de Carbono (endógeno) funciona como neurotransmissor. Actua difundindo-se entre neurónios e entre estes e attras células do organismo.

A difusão do CO do ambiente para o sangue é muito rápida: o co inalado difunde-se através das paredes alveolares ligando-se ao anel da hemoporfirina da hemoglobina e, também, à mioglobina (Strav, Mbooy, Jorns, 2006).

A ligação do Monóxido de Carbono com a hemoglobina, pigmento vermelho do sangue que transporta o Oxigénio, é uma ligação de "longa duração", formando carboxi-hemoglobina, mesmo em exposição por curtos períodas de tempo. De realçar que a afinidade do co para a hemoglobina é cerca de 200 a 250 vezes maior que a do O2, existindo contudo variação de indivíduo para indivíduo. Relativamente à citocrono Coxidase, pelo contrário, a afinidade para o $\mathrm{O}_{2}$ é 10 a 20 vezes superior à do CO (Ciark e Thom, 2004). 
A captação apresenta características muito próprias, pois é exponencial, sendo factores determinantes a frequência ventilatória e a profundidade da vent i lação pulmonar, a concentração de co no "ar" inspirado, a duração da exposição.

A dissociação da carboxi-hemoglobina varia de acordo com a hemoproteína a que o co se ligou; da quantidade de hemoglobina "incapacitada" vão resultar sintomas de falta de "oxigénio" apesar de a vítima se encontrar em ambiente em que este abunde, pois na realidade trata-se de uma "asfixia ao nível das tecidas".

A afinidade entre o co e as citocramos, proteínas com um grupo "heme", contendo ferro, as quais fazem parte da cadeia de transporte de electrões igualmente designada por cadeia respiratória, ligada à respiração aeróbica, encontra-se relacionada com o mecanismo de actuação do tóxico. Impõe-se afastar imediatamente a vítima do local e iniciar o tratamento.

A semi-vida da carboxi-hemoglobina é de 5 a 6 horas a 1 atmosfera quando a vítima respira ar. Se a vítima for imediatamente colocada a respirar Oxigénio $\left(\mathrm{O}_{2}\right)$ a 15 litros por minuto, a 100\%, à pressão atmosférica "hormal" (1 atmosfera) a semi-vida passa a ser de 90 minutos. Com o Oxigenio Hiperbárico (3 atmosferas) reduz-se para 23 minutos (NAVARRO e Ruco, 2007) . Por outro lado a quantidade de $\mathrm{O}_{2}$ dissolvido no sangue passa a ser cerca de 10 vezes mais elevada (Hornan et al., 2007).

Daí, ser fundamental ter procedimentos de intervenção, na luta contra o fogo e autras situaçães em que exista o risco de intoxicação pelo Monóxido de Carbono, de modb a ser possível manter a vítima a respirar $\mathrm{O}_{2}$ purro até à chegada à Câmara Hiperbárica Terapêticica.

Aos indivíduos com patologia pulmonar, nomeadamente com passado tuberculoso, e às grávidas deve ser prestada atenção especial, pois apresentam risco de morbilidade e mortalidade acrescidas. No caso da grávida o factor fundamental a corrigir é a hipoxia fetal. Estudbs efectuados revelam que o tratamento com oxigénio hiperbárico é seguro na gravidez (Hofrven, et al., 2007) .

O tratamento com Oxigénio Hiperbárico, em Portugal, encontra-se, actualmente, disponível em Lisboa, noHbspital da Merinha (pioneiro em Portugal) , na Horta, nos Açores e, recentemente, emMatosinhos, no Hospital Pedro Hispano. De acordo com a Comunicação Social deverá seguir-se a Madeira.

A possibilidade de efectuar a monitorização do Monóxido de Carbono nos intervenientes com equipamentos de fácil uti lização presentemente não é consensual segundo alguns autores (CLARK e Trov, 2004) . Porém existe investigação em arso sobre novas tecnologias neste ânbito.
Deve ser relevadb o facto de ser possível virema surgir consequências tardias da intoxicação pelo Monóxido de Carbono (associada com perda de consciência na fase aguda) : cerca de 50\% dos doentes com intoxicação aguda sintomática ficam com sequelas cognitivas. Parecendo inicialmente ter existido "restitutio ad integnum" com umperíodb de lucidez que poole ir de 2 a 40 dias após intoxicação inicial por co podem surgir: demência, síndromes amésicos, psicose, Parkinsonismo, paralisia, coreia, cegueira cortical, apraxia, agnosias, neuropatia periférica e incontinência. Doentes com idade superior a 30 anos parecem ser muito mais susceptíveis de desenvolver sequelas tardias (HHFMN et al. , 2007).

A "Profissão" de Bombeiro continua, contudo, esquecida na actualizadíssima Lista das Doenças Profissionais.

No Diário da República da I Série, número 136, de 17 de Julho de 2007, páginas 4499 a 4543 foi publicado o Decreto Regulamentar $n^{\circ}$. 76/2007, que procede à alteração dos capítulos $3^{\circ}$ e $4^{\circ}$ da Lista das Doenças Profissionais publicada em anexo ao Decreto Regulamentar nº 6/2001 de 05 de Maio.

Em anexo a este novo Decreto Regulamentar 76/ 2007 é publicada a Lista das Doenças Profissionais actual izada - republicação do Decreto Regulamentar $n^{\circ}$. 6/2001 de 05 de Maio.

No Capítulo I, sob o Código 11.10 - Óxido de Carbono (Monóxido de Carbono) , o trabalho dos Bombeiros continua, lamentavelmente, a não constar da. "Lista exempl ificativa dos trabalhos susceptíveis de provocar a doença" .

Deve ser ainda enumerada autro tipo de patologias que não só os bombeiros mas também as vitimas podem apresentar por exemplo, em incêndios florestais, encaradbs numa perspectiva de intervenção em Medicina de Catástrofe como seja: Patologia ocular, Cutânea, Traumatológica, Respiratória, Cardiovascular, Electrotraumatismos, Golpes de Calor, Crises de Epilepasia, etc. não devendo nunca ser esquecidas as nomas higiénico dietéticas relativas à alimentação, nestas situações.

A protecção ao Bombeiro e a respectiva padronização do Equipamento, quer em situação de socorro à univitíma quer em situação de Catástrofe, encontra-se devidamente padronizada na nossa vizinha Espanha, através da "Guia de Selección de Equipos de Protección Individual" da Asepal (2002) . Trata-se de um trabalho de referência e de extraordinária importância que está a ser estudado por uma das nossas alunas do Mestrado em Medicina de Catástrofe, com vista à elaboração da sua Dissertação. 


\section{Intervenção no Terreno}

O interesse mundial em lidar com situaçães de catástrofe e o desenvolvimento tecnológico desencadeou novas abordagens na avaliação de situações de emergência, triagem e tratamento no ambiente pré-hospitalar e hospitalar (GuI, 2006) . A capacidade de trazer rapidamente experientes "handson", físicas e tecnológicas, en situações de doentes politraumatizados permitiu aproximar a tecnologia ao desenvolvimento real no terreno.

Actualmente o equipamento de imagem utilizado num hospital de campanha, nomeadamente no Afeganistão, representa tecnologia inovadbra numca utilizada num ambiente hastil por longos períodbs. O facto de terem acesso a aparel hos de Tomografia Computorizada (TC) e Ultrassonografia (US) juntamente com o equipamento de Radiologia Convencional (Rx), foi condição fundamental na avaliação e orientação terapêutica dos doentes, tornando-se uma prioridade que um médico especialista em imagem seja enviado para o hospital de campanha, particularmente quando um sistema de TC e US fizerem parte do equipamento. Gry et al., (2006) no seu relatório acerca da experiência no Afeganistão da presença da Imagem num ambiente hostil fornece à comunidade médica importante informação na orientação, planeamento e funcionamento de equipamento que incluía imagem digital no campo de batalha.

Madame Arie (1867-1934) referia, no seu livro, "La Radiologie et la Guerre", que "a história da radiologia na guerra oferece impressionante exemplo da insuspeitada amplitude que, em certas condições, pode tomar uma descoberta de ordem puramente cietúfica."

Todos as anos muito desastres causam milhares de lesões, mortes, refugiados, etc.... Terramotos, guerras e outras situações de catástrofe originam lesões severas, por ex: queimaduras, amputações, feridas por armas de fogo, minas, material nuclear, biológico, químico, infeccioso (Baner, Schatmorr, NERLICH, 2004).

Nesta era de terrorismo gldbal, cenários de guerra e situações de catástrofe de etiologia variada, particularmente desde o 11 de Setembro de 2001, as fronteiras entre lesões traumáticas militares e civis foram atenuadas (BसkNEN, et al., 2007) . Assim sendb, a Telemedicina, definida pela Organização Mundial de Saúde (OMS, 1997) como a prestação de serviços ligados aos cuidados de saúde nos casos em que a distância é um factor crítico, veio revolucionar opapel da imagem nessas situações disponibilizando o acesso em tempo útil ao diagnostico imagiológico dos doentes o que permite assim uma adequada avaliação e posterior orientação.
Tais serviços são prestados por profissionais da área da saúde, usando tecnologias de informação e de comunicação visando o intercâmbio de informações válidas para o diagnóstico, prevenção e tratamento de doenças e a educação contímua dos prestadores de cuidados de saúde, assim como para fins de pesquisas e avaliaçães, com o dbjectivo de melhorar a saúde das pessoas e de suas comnidades.

A abordagem das lesões traumáticas craneo-

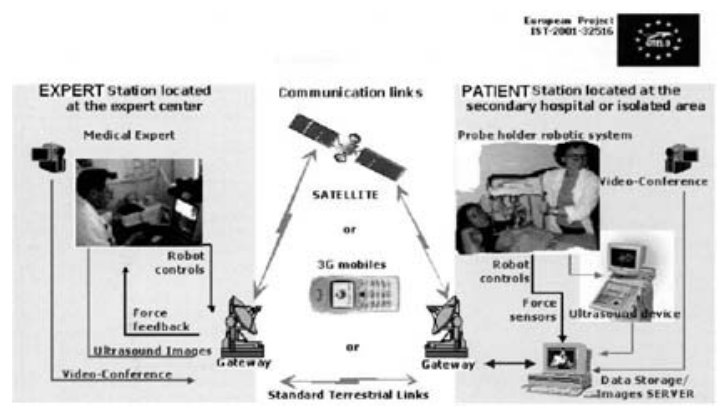

Fig. 1 - Sistema Otelo (Carkzs et al., 2005) .

encefálicas, vertebro-medulares au toraco-abobminais foram sempre um desafio para os médicos em geral, militares au civis. Durante o séc. XX os avanços técnicos permitiram aos cinurgiões centrarem-se na correcção de lesões vasculares com revascularização, restauração da perfusão e a consequente preservação de membros e da vida (Wertr et al., 2006) tendo tido as cinurgiões militares portugueses, camo Reynaldo dos Santos, um papel fundamental. Para além da descoberta e desenvolvimento da aortografia e angiografia arterial dos membros o que veio corroborar o papel fundamental da imagem na aplicação médica, teve amplas contribuições no âmbito da cinurgia em geral e cinurgia vascular em particular com relevo para o desenvolvimento de técnicas cinúrgicas inovadoras em situação de Guerra.

No âmbito da Medicina de Catástrofe (BANEIRA, 1995) , in latu senso, a imagem assumirá um papel cada vez mais preponderante. Desde a intervenção no terreno, com as viaturas radiológicas da Mbdame Arie até ao momento presente, ande os equipamentos de imagem e respectivos especialistas podem actuar quer a nível dos PMA (posto médico avançado), CME (centro médico de evacuações) e HR (hospital de retaguarda) , não só autonomamente mas igualmente utilizando a Telemedicina numa articulação de meios cada vez mais sofisticados. combase em equipamentos fiáveis tecnologicamente, que cada vez mais ocupam menos espaço físico, apresentam maior mobilidade e concomitantemente podem ser deslocadbs commaior facilidade ao teatro de operaçães. 
Os dbentes, poderão assim, beneficiar desta área de especialização médica que apresenta cada vez maior evidência. Apresentamos, pois, exemplos de alguns doentes vítimas de traumatismos craneoencefálicas, vertebro-medulares e toraco-abolominais e as diversas técnicas de imagem ( UltrassonografiaUS, Tomografia Computorizada - TC e Ressonância Magnética - RM) com as suas diferentes aplicações, indicações e informações:

- Doente com traumatismo toraco-abdominal cujo exame de US mostra a presença de um derrame pleural/ hemotorax (1) .

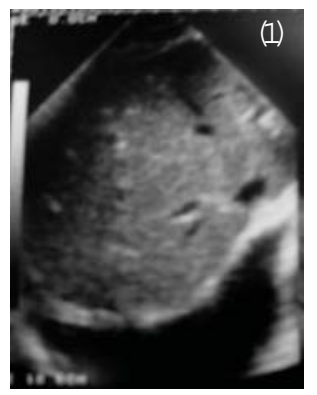

- Doente vítima de traumatismo craneo-encefálico no qual o exame de TC mostra a existência de um hematoma subotural, que exerce marcado efeito de massa sobre o parênquima cerebral subjacente, condicionando edema cerebral e herniação subfalcial (2a e 2b) .
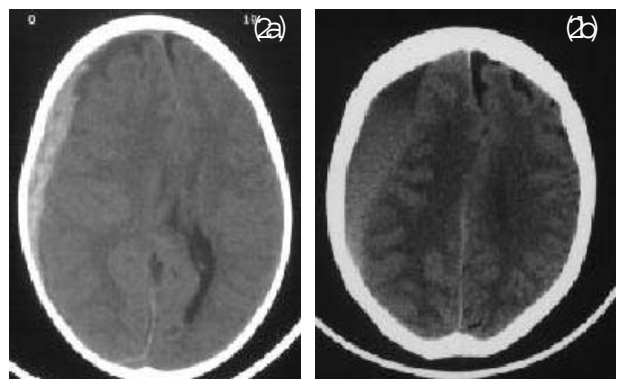

- Doente com traumatismo vertebro-medular pós queda, tetraparético, com indicação formal de real ização de exame de RM que mostrou sinais de fractura/luxação C6-C7 com franca compressão do cordão medular e sinais de sofrimento medular (edema) (3a e 3b) .
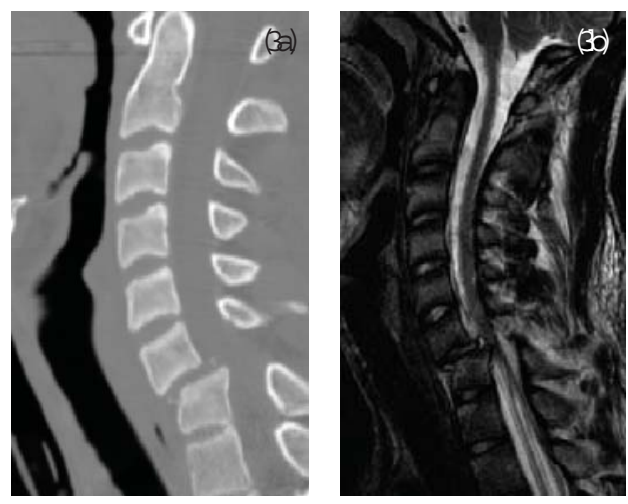

Os doentes vítimas de traumatismos toracoabodominais podem sofrer múltiplas lacerações de vários órgãos como pulmões, fígado, rins e baço, com consequentes lesões hemorrágicas associadas (4) .
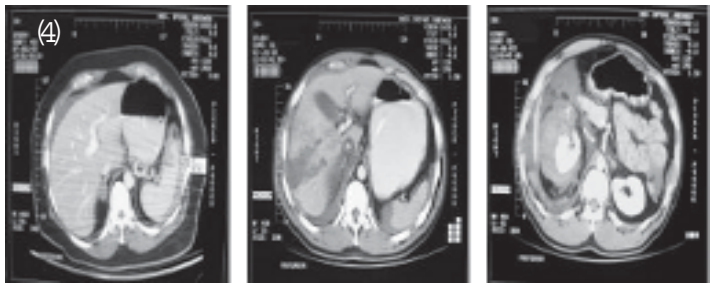

Lesões cerebrais e cervicais são frequentes em doentes vítimas dos mais variados tipos de traumatismos condição que tem vindo progressivamente a aumentar, tendo sido por isso necessário desenvolver o mais variado tipo de equipamento de protecção individual, de que são exemplo capacetes, óculos, coletes de protecção, etc..

Com a capacidade de evacuação rápida desde o terreno, por via aérea, levantam-se alguns aiidados a ter com as doentes, nomeadamente vítimas de lesões do seio frontal. Estes doentes podem sofrer mudanças barométricas importantes condição que pode ser evitada com diagnostico atempado e correcto transporte e abordagem terapêutica para prevenir lesão secundária, como aumento da pressão intracraniana ou pneumbencefalocelo de tensão o que iria prejudicar a abordagem do dbente no nível seguinte de terapêutica. Os princípios da abordagem como a importância do baixo nível de voo /pressurização da cabine uso de descongestionantes, evitar mandora de Valsava, e lidar com possíveis complicações na aterragem. Preconiza-se nestes casos um mecanismo simples de equilíbrio da pressão em doentes com compromisso do seio frontal durante uma evacuação aérea usando um angiocateter colocado na ferida antes da sutura.

Exemplo de exames de Radiologia Convencional (Rx) , Tomografia Computorizada (TC) e Ressonância Magnética (RM) em doente com traumatismo craneoencefálico, apresentandb vários traços de fractura na região frontal: há hemiação do parênquima encefálico à direita e hematomas subourrais bihemisféricos (5) .

\section{Imagem versus Intoxicação por co}

Um quadro de encefalopatia anoxico -isquémica origina lesões cerebrais bilaterais causadas pela inalação de co e falta de perfusão cerebral. A intoxicação por Monóxido de Carbono leva caracteristicamente a necrose do gldous pall idus que consiste em hiperintensidade central na região palidal em T2, ou hipodensidade em TC, sugerindo edema citotóxico, e lesões na substância branca dos hemisférios cerebrais (OBRRE, 2004). 

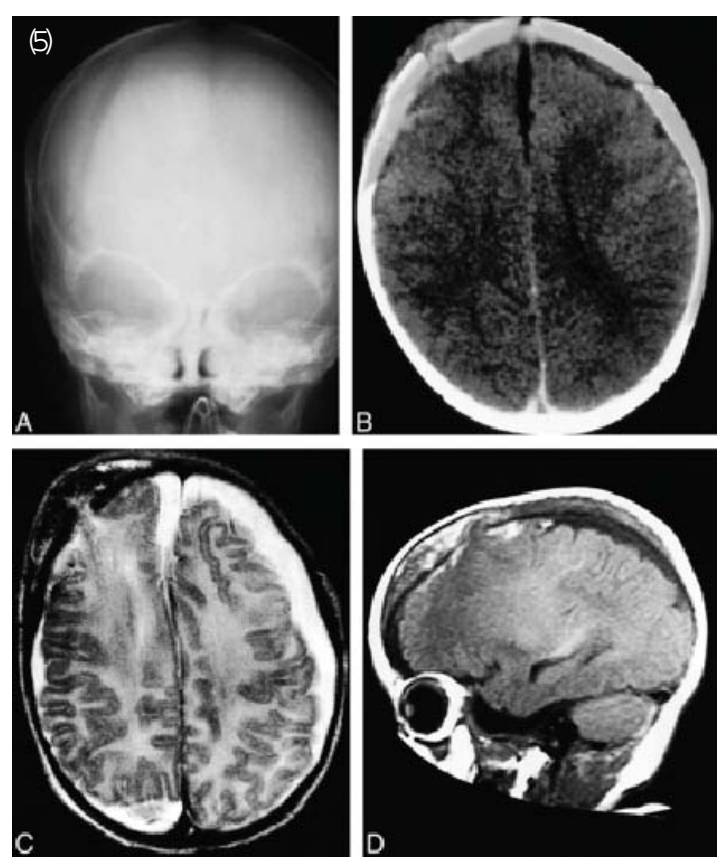

Doentes com lesões desta natureza deverão ser avaliados no contexto clinico-patológico e estabelecido diagnóstico diferencial com lesões por intoxicação por barbitúricas, cianeto, sulfato de hidrogénio ou hipotensão. As lesões por hipoxia podem também envolver os hipocampos cujos neurónios são vulneráveis às lesões por anoxia.

Exemplo de doente com quadro de encefalopatia anóxico-isquémica em cona: lesões das núcleas da base e atingimento bi lateral da substância branca (6a e 6b) .
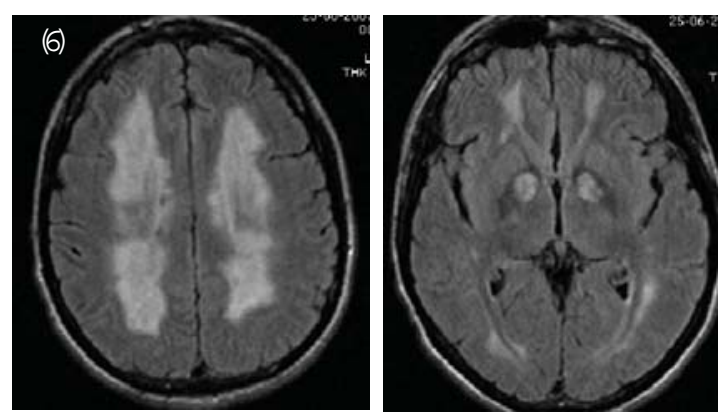

\section{Formação}

Em recente trabalho publicado na Revista da Federação dos Bombeiros do Distrito do Porto, (GAnRRA, 2007) chamamos a atenção para a ênfase que foi posta no seguinte período do mesmo "a aquisição de conhecimentos quer científicos quer práticas que as elementas dos Conpas de Bambeiros, que posteriormente venham a integrar as equipas de intervenção, tem que dbrigatoriamente ser adquiridos através de formação e instrução contínuas nas mais diversas áreas de intervenção" .
A formação e o empenhamento a que ela doriga, fez com que o antigo Serviço Nacional de Bombeiros, através de protocolo com o Instituto de Ciências Biomédicas de Abel Salazar (ICBAS), estivesse representado por cinco bolseiros, no Mestrado em Medicina de Catástrofe, dois dos quais, o Mestre António Gomes que realizou a sua dissertação, intitulada "Formação do Bombeiro em Saúde em contexto de ACFI - Distrito de Aveiro" e a Mestre Sara Gandra, que igualmente fez a defesa da sua Tese intitulada, "Importância da Medicina Legal em Situação de Catástrofe - A Queda da Ponte Hintze Ribeiro", ambas tendb dotidb a classificação de Muito Bom por unanimidade, colocaram assim a tónica em duas das áreas do Serviço de Saúde, designadamente na da Formação e na de Intervenção, sendo doras de consulta e referência dorigatórias.

À Escola Nacional de Bombeiros consideramo-la como um baluarte no ensino de matérias no domínio do Socorro; trata-se pois de uma Instituição incontomável e que em recente resposta a inquérito atinente à preparação de uma dissertação de doutoramento escrevemos que a ENB poderá e deverá ter um Centro Nacional Especializado e Coordenador (ANEC - Sintra) , existem Centros de Formação Geral Regionais (CFGR), 6 com alguns componentes específicos de acordo com o mapa de riscos, designadamente em Porto/Braga, Vila Real/ Bragança, Coimbra, Covilhã/Guarda, Évora e Algarve. Para além destes centros intermediários a formação continua e de base deveria ser feita nos corpos de Bombeiros quer pelos formadores intemas, credenciados pela ENB, quer por formadores dos aludidos CFGR que se deslocariam aos CB, a fim de colmatarem as lacunas existentes.

Não nos podemos de forma alguma esquecer que no âmbito dos voluntários, a formação deve ir aos mesmos e não aqueles a esta, uma vez que não se trata de profissionais que hoje e cada vez mais não podem pôr em causa a sua Família e os seus postos de trabalho.

\section{Canclusões}

Através dos dados estatísticas que a seguir se publicam por amável deferência da Direcção da Escola Nacional de Bombeiros (ENB), podemos aquilatar da importância da acção dos Bombeiros, face à exemplaridade do trabal ho desenvolvido.

Não podemos esquecer, de acordo com Kares et al., (2007) que "A death was classified as being associated with fire suppression if it ocanred while the personwas fighting a fire or at the scene a fire after its suppression. Alarm response involved responses to emergency incidents, including false alarms." 
Quadro 1- Corpos de Bombeiros Ponto de Situação Nacional Serviços Prestados de 1/1 a 31/12/2006. (Excepto Transporte de Doentes)

\begin{tabular}{|c|c|c|}
\hline \multicolumn{2}{|c|}{} & $\%$ \\
\hline 1-INCÉNDIOS & 47.768 & 5,3 \\
\hline 2-ACIDENTES & 50.688 & 5,6 \\
\hline 3. OCORRENCIAS DIVERSAS & 24.109 & 2,6 \\
\hline 4. SERVIÇOS DE APOIO & 120.585 & 13,3 \\
\hline 5 - PRÉ-HOSPITALAR & 657.899 & 73,2 \\
\hline 6 - ACTIVIDADES FORMATIVAS & SUBTOTAL & 901.049 \\
\hline 7. FALSOS ALARMES & & 12.912 \\
\hline & & 146.162 \\
\hline & TOTAL & 1.060 .123 \\
\hline
\end{tabular}

O Serviço de Saúde não pode nem deve continuar a actuar de uma forma velada, diríamos mesmo como que "envergonhada". Obrigatoriamente dever-se-á alicerçar e projectar independentemente de todas as acções que nesta área já foram ou venham a ser pontualmente desencadeadas com todo o mérito por outras entidades, uma vez que é detentor de uma filosofia especial e de um savoir faireque lhe permite uma acção ímpar a todos as níveis.

\section{Bibliografia}

Acostrint, E. (1995) - "Desenvolvimento do Serviço de Saúde nos Bambeiras Portugueses" . in: Bambeiros Portugueses - Seis séculos de História 1395-1995, pp. 43-60 vol. $1 \mathrm{Ed}$. SNB/LBP Lisboa.

ASEPAL (2002) - Guia de Selección de Equipos de Protección Individual. Asociación de Empresas de Equipos de Protección Personal, Madrid.

BANDERA, R. (1995) - Medicina de Catástrofe. Da Exemplificação Histórica à Iatroética, Tese de doutoramento, ICBAS, Universidade do Porto.

Breckey, A. C., STARRES, B.W., StresTA, J. A. (2007) Lessions leamed frommodem military surgery, Surg. Clin. North. Am. Fev.; 87 (1) :157-84, vii.

BENNET, T.; SCHACHINGER, U. ; NERLICH, M. (2004) Telemedicine in trauma and disasters - from war to earthquake: are we ready?, StudHealth Tednol Inform, 104: 106-15.

Bryson, B. (2005) - A Short History of Nearly Everything - Illustrated, Doubleday, Transworld Rublishers, Iondon.

Carardg, G. (2004) - Mbnáxido de Carbono. In: Cañadas E. V. (ed) Calaburig - Nedicina Legal YToxicología, $6^{2}$ ed, Masson SA, Barcelona, pp 829 - 835.
CAFK, J.; Trm, S. (2004) - Toxicity of Oxygen, Carbon Dioxide and Carbon Mbnoxide. In: Bove AA (ed) , Bove andDavis' Diving Medicine, 4thed, Saunders, Elsevier Inc. , Philadelphia, pp 241-259.

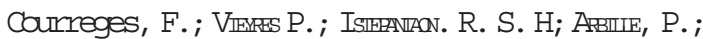
Bro, C. ; (2005) - "Clinical Trial and Evaluation of a Mbbile Robotic Tele-ultrason System", Joumal of Telemedicine and Telecare 11, Supl. 1: 46-49.

CRistiano-Saños (1995) - "Evolução da Organização conporacional". in: Bambeiros Portugueses - Seis séculos de História 1395-1995, pp. 211-218 vol. 1 Ed. SNB/LBP Lisboa.

CRRE EtA - Madame Curie, $2^{a}$ edição, colecção Dois Mundos, tradução Mbonteiro Ldbato, Revisão Freitas Leça , Edição Liviros do Brasil, Lisbooa, pág 210-223.

Franç, G.V. (2004) - Medicina Legal, $7^{a}$ ed, Guanabara Koogan, Rio de Janeiro.

Gill, R. (2006) - "Irnovationpossibilities forprehospital providers", Prehosp. Energ. Care, Jil-Sep;10 (3) : 317-9.

GيnRA, R. (2007) - "Quem não sabe, não salva, nem se salva" . Rev. Federação dos Bambeiros do Distrito do Porto. Ano 1, n. ${ }^{\circ} 0: 7$.

GandA, S. (2005) - Importância da Medicina Legal em Situação de Catástrofe - A queda da Ponte Hintz Ribeiro - Dissertação de Mestrado. ICBAS Porto.

GHE, H. ; HFRE, H. T. ; STHIHR, J. D. ; MbIIIA, J. (2006) - Radiology in a hostile envirament: experience in Afghanistan, Mil Med, 171 ( 3) : 194-9.

Goves, A. (2004) - Formação do Bombeiro em Saúde em contexto de ACEL. Dissertação de Mestrado. ICBAS Porto.

Hoffman, R.; Netson, L.; Howland, M. ; LewIN, N. ; Frovenaaim, N. ; Godprrancr, L. (2007) - Goldfranks's Merual of Toxicologic Energencies, The MoGraw-Hill Companies, Inc. New York.

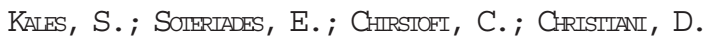
(2007) - "Emergency Duties and Deaths from Heart Disease amang Firefighters in the United States". The New England Joumal of Medicine, Vol. 356, n. ${ }^{\circ} 12$ : 1207-1287.

Navarro, W. ; Rugo, H. (2007) - Occupational Hematology. In: LaDou J. (ed) Occupational and Envirormental Medicine, 4th ed, McHill, New York, pp 217. 
OARRE, A. (2004) - Diagnostic Imaging Brain. Elservier Saunders Amirsys. Salt Iake City.

Simav, B.; Mbor, E.; Jans, R. ; (2006) - Therapeutic Gases - Oxygen, Carbon Dioxide, Nitric Oxyde and Helium. InBrunton L, Lazo J, Parker K (eds) - Goodman and Gilman's The Pharmacological Basis of Therapeutics, $11^{a}$ ed, MbGraw-Hill, New York pp 387399.

WeArR, M. A. ; FOX, C. J. ; ADAN, E., RTCE, R.D. ; Q Q Cox, M.W. ; GMHESPE, D.L. (2006) - "Upper extremities arterial combat injury management", Perspect. Vasc. Surg. Fnobvasc. Ther. Jun ; 18(2):141-5.

\section{Anexos:}

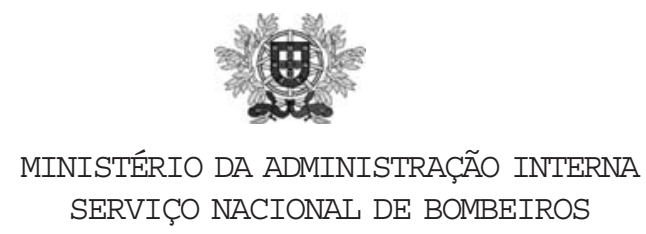

\section{DESPACHO}

A Inspecção Regional de Bombeiros do Norte, recentemente instalada, ainda não dispõe de pessoal de apoio para além do inspector.

As tarefas que lhe estão cometidas requerem, em algumas áreas, designadamente no sector de saúde, a colaboração de pessoal especializado, habilitado a pronunciar-se acerca das mais diversas questões entre as quais se salientam os aspectos ligados à formação e informação dos elementos das conpos de bombeiros e respectivo conteúado.

Contactados diversos elementos considerados capazes de desempenharem a missão foi escolhido o Dr. Romero Manuel Bandeira Gandra por ser o que oferecia melhores condições.

Lisboa, 30 de Setembro de 1982

O PRESIDENIE DA DIRECÇÃO

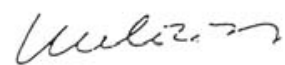

V. J. Melícias Iopes

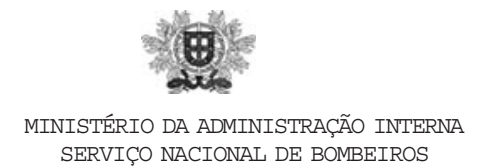

ASSUNTO: Estudo para a Implantação e Funcionamento de um Departamento de Saúde ao nível do SNB.

São as corporaçães de Bombeiros os Órgãos de Socorro e contacto directo com as populações ao nível dos Municípios e aquelas que verdadeiramente desenvolvem os seus serviços consoante as reais necessidades das populações que servem. Assim, e ao longo do tempo elas viram nascer e desenvolver as Serviças de Fogo - a que deveram o seu name - os Serviças de Saúde e as Socorros a Náufragos se porventura a Zona de actuação era marítima ou fluvial.

Conclui-se, daservandb factos evidentes, que é sem dúvida o Serviço de Saúde dos corpos de Bombeiros aquele que apresenta maior expressão.

\section{B - OBJECTIVOS A ATINGIR}

Como só com a dignificação da função se conseguirá o respeito, a admiração, em suma, a confiança do Público, seja ele diferenciadb au não, deveria o Departamento de Saúde do SNB estar vocacionado para:

B.1. Boa preparação das Equipas de Socorro, quer no plano técnico, quer no dentológico/Formação Nacianal (ver anexos I, II, III arsos) .

B.2. Capacidade de atendimento em qualquer situação de emergência.

B.3. Estudar profundamente a Metodologia dos Transportes não urgentes:

B.3.1. Transportes de rotina;

B.3.2. Transportes com cuidados medicalizados ou não.

B.4. Correcta e completa informação do público e tanto quanto possível, na formação a nível local/muicipal.

B.5. A nível de Região a acção do Departamento de Saúde das Inspecções crientar-se-iapara:

B.5.1. Ministração dos arssos conscante os níveis a toob o pessoal dos Corpos de Bombeiros;

B.5.2. Pareceres técnicos sobre viaturas e equipamentos inseridas na área da saúde;

B. 5.3. Juntas Médicas;

B.5.3.1. Junta de Recnutamento para todb o pessoal a adnitir, inclusive os elementos do camando.

- O padrão a seguir seria, por exemplo, o sistema SICVAGE, utilizado na selecção de pessoal para as Forcas Armadas, bem camo o uso respectivo da tabela de lesões. (ver anexo IV, tabela de lesões e limites de idades) .

B.5.3 .2 . Juntas Médicas para promoção ao posto imediato, bem como ao pessoal oriundo do Quadro de Inactividades (Dentro e Fora Q) .

B.5.3.3. Juntas Médicas para Motoristas dos Conpas de Bambeiros segundo os parâmetros usados para Instnutores de Condução pela Direcção Geral de Saúde.

B.5.3.4. Nas Inspecções Médicas seria dorigatório:

Nos outros Casos $\mid$\begin{tabular}{l|l} 
SICVAGE \\
MRX \\
BIV (Boletimintes Individal de Vacinas) \\
Análises Clínicas (Hemograma + V.S. Ureia \\
+ Creatinémia + Uricemia + Glicemia + \\
TGP+TGO sumária de Urina.
\end{tabular}

$\mathrm{ECG}+\mathrm{Rx}$ Pulmonar + outros dados completos se necessário.

B.5.3.5. Do resultado das Juntas médicas não haveria rearrso. B.5.3.6. A Juntas Médicas efectuar-se-iamas Inspecções Regianais. 
- Dignificação;

- Igualdade na doservação;

- Não sujeição à influência local.

- Ficheiro organizado e possibilidade de comparação

de dados imediatamente para toda a Região.

B.5.4. Inspecções sanitárias aos Quartéis e demais Instalaç̃̃es dependentes. Seguindo as normas de salubridade da D.G.S. incluindo o Regulamento da Ed. Unbanas.

\section{C. - ORGÃOS E FUNCIONAMENTO}

C.1. Ao SNB, através do Departamento Central de Saúde, realmente descentral izado no Departamento de Saúde das Inspeccõões, mas sempre sob a tutela do Director de Serviços Técnicos, campetiria:

C.1.1. Aprovar a acção Regional a nível das Inspecções.

C.1.2. Contactar, sempre que necessário, organismos vários, nomeadamente:

Faculdade de Medicina, Hospitais Idóneos onde se professam especialidades que digam respeito às tarefas exeatadas por este Departamento, Ordem das Médicos, Sindicato das Enfermeiros, etc. C.1.3. Criação de uma Biblioteca Especializada.

C.1.4. Contacto cam centros especializados no estrangeiro.

C.1.5. Montar secção de Textos de Apoio.

C.1.6. Adquirir Filmes, Slides e autro material didáctico julgado necessário.

C.2. Criar junto do Departamento Central um Núcleo de Médicos Consultores, baseado nos Colégios de Especialidades da Ordem dos MÉdicos, especialmente nas seguintes especialidades: Cinurgia Geral, Medicina Interna, Anestesias/Reanimação, Cardiologia, Ortotraumatologia, Costetrícia.

Se passível todbs as técnicas deveriam ser escolhidas dentro do núcleo de pessoas que esteja ou tenham estado reconhecidamente ligados aos Bambeiras.

\section{ESTRUIURA A NÍVEL DE PESSOAL}

Dada a acção diversificada a real izar na área da saúde, preconizada acima, como sejam: os pareceres técricos, as Juntas de Recrutamento, Formação de Pessoal e a permanente acção junto dos Corpos de Banbeiros, entende-se necessária a seguinte estnutura:

D.1. Cam o fim de avançar rapidamente, criar, na dependência directa db director dos Servicoss Técnicos, dbis assessores Técnicos Licenciados em Medicina cam funções de:

a) apoio à coordenação dos Serviços de saúde;

b) orientação na acção dos médicoss do D.S.I.R. ;

c) Integrar e presidir às Juntas sempre que se julgue necessário;

d) Formação de Pessoal.

e) Atras quaisquer funções que se venham a tomar pertinentes.

Destes dois assessores, um exercerá a sua função nas áreas abrangidas pelas Inspecções Norte e Centro e o autro nas áreas das Inspecções de Lisboa, Alentejo e Algarve.

D.2. Nas áreas de cada Inspecção Regicnal existência de um médico dependente da DST e responsável pelo DSIR tendo as funções de orientação e formação de pessoal, integração de Juntas Médicas, Inspecções Sanitárias a Quartéis, bem como fomecer aos Inspectores os pareceres técricos que aqueles lhes venhama solicitar.

D.3. En cada distrito o DSIR contará pelo menos com 2 instrutores, cujas habilitações académicas mínimas serão o Curso de Enfermagem e que deverão vir a possuir a especialidade de enfermagem de emergência (Grau III).

D.4. Nos Corpas de Bambeiros existirão 2 graus:

I - Para Banbeiros;

II - Ctes., Ajuates. Chefes e Subchefes.

D.4.1. Năo é impeditivo, muito pelo contrário, que qualquer elemento do corpo de Bombeiros venha a adquirir, ou até já possua, um grau superior ao que legalmente lhe campete.

D.4.2. Pretende-se que os Corpos de Bombeiros estejam em aperfeiçcamento permanente, devendo os elementos de grau II assegurar-se de que os de Grau I estejam actual izados e doviamente a DSIR fará as reciclagens necessárias para uma permanente qperacionalidade.
D.5. Os exames serão efectuados por um júri constituŕdo sempre por dois elementos de um grau imediatamente superior ao examinando e o attro de grau superior ao $1^{\circ}$ examinador: presidirá o mais qual ificadb. D.6. Todos as actuais Instrutores e Mbnitores serão integrados nas funções para que estão preparados depois de aval iadb o seu Curriculum pelo DS Central.

Dr. Ramero Bandeira

Dr. Eduardo Agostinho

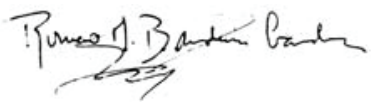

TABELAS DE LESÕES PARA USO DAS JUNTAS DE RECRUTAMENTO DE BOMBEIROS

CAPÍTULO I

1. Aplicação das Tabelas

Tabela 0 - Para candidatos a Aspirantes e Bambeiros de $3^{\text {a }}$ classe.

Tabela A - Para Comandantes, Ajudantes e indivíduos oriundas dos quadros de inactividade com idade superior a 35 anos.

Tabela B - Para indivíduos oriundas das quadros de inactividade com idade inferior 35 anos.

Tabela M - Para banbeiros abrangidas por disposições semelhantes às do Dec. Lei 210/ 73 de 9 de Maio (a adoptar) .

\section{Legenda das Tabelas}

AD - A aguardar confirmação da aptidão.

+ - Inapto, isento au incapaz de todb o serviço au do serviço activo.

+ - Inapto quando nas condições expressas na tabela; apto para serviços auxi liares, apto para serviços moderados au pranto para todb o serviço, se natras candiçães.

H+ - Apto para servicos auxiliares.

+1+ - Apto para o desempenho de cargos ou funções que dispensam plena validez (Dec. -Lei n' 210/73 a adaptar) .

(-) - Pronto para todb o serviço au apto para todb o serviço activo (Dec. -Lei $n^{\circ} 210 / 73$ a adaptar)

Conforme critério da junta e grau de lesões.

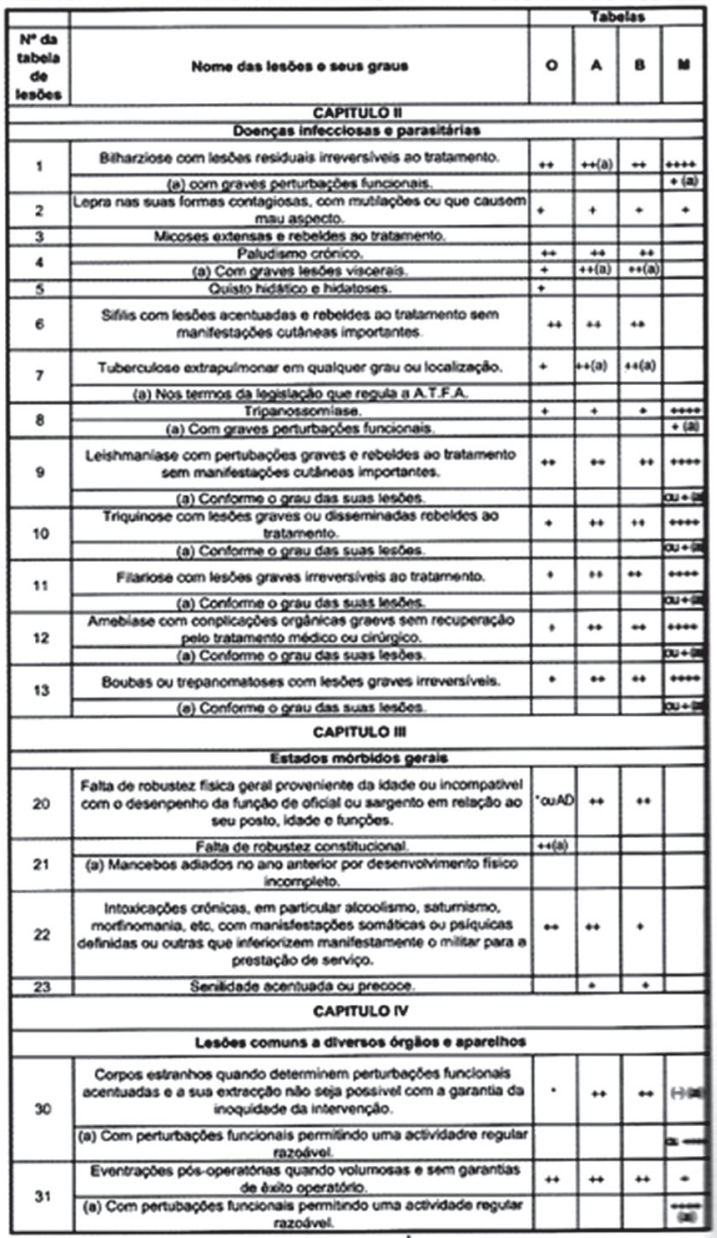


(Página deixada propositadamente em branco) 


\section{Fernando Rebelo}

Nota de Abertura

\section{João Figueira}

- "Uma união de factos contemporânea: Jornalismo e situações de risco"

\section{Maria João Silveirinha}

- "A vida no arame. A mediatização do risco".

\section{Fantina Tedim, João Gonçalves}

- "Simulation of the 1755 tsunami flooding area in the Algarve (Southern Portugal): the case-study of Portimão"

\section{António S. Pedrosa, B. S. Marques, B. Martins, J. H. Sousa}

- "Quaternary evolution of the Serra do Marão and its consequences in the present dynamics"

\section{Andreia Pereira, António S. Pedrosa}

- "Paisagem cultural das montanhas do Noroeste de Portugal: Um ciclo de construção, desestruturação e reconversão"

\section{Fernando Rebelo}

- "O risco de sedimentação na laguna de Aveiro: Leitura actual de um texto de Amorim Girão (1922)"

\section{Fantina Tedim, Maria Lúcia de Paula Herrmann}

- "A comparative analysis of forest fire policies in protected areas in Portugal and in the state of Santa Catarina (Brazil): a general approach"

\section{António Bento Gonçalves, Luciano Lourenço, João Dias}

- "Manifestação do risco de incêndio florestal. Causas e investigação criminal"

\section{Lúcio Meneses de Almeida}

- "Comunicação do risco e gestão da ameaça pandémica"

\section{Romero Bandeira, Ana Mafalda Reis, Rui Ponce Leão,} Sara Gandra, Romero Gandra

- "O serviço de saúde nos bombeiros. Sua importância, da Univítima

à Medicina de Catástrofe"

Notas, notícias e recensões

\section{Luciano Lourenço}

- "Riscos naturais, antrópicos e mistos"

\section{Gisela Oliveira}

- "Comunicar numa situação de emergência ou de crise"

\section{Luís Manuel Guerra Neri}

- Curso sobre Catástrofes Naturais.

\section{Fernando Rebelo}

- Socalcos e Riscos Naturais estudados no âmbito do Projecto Europeu TERRISC

\section{Fernando Rebelo}

- Incêndios Florestais e suas consequências discutidos nas VI Jornadas Nacionais do PROSEPE 